\title{
Educational Context of Mathability
}

\section{Katarzyna Chmielewska}

Institute of Mathematics, Kazimierz Wielki University, Chodkiewicza 30, 85-064, Bydgoszcz, Poland, katarzyna.chmielewska@ukw.edu.pl

\section{Attila Gilanyi}

Faculty of Informatics, University of Debrecen, Egyetem tér 1, 4032 Debrecen, Hungary, gilanyi.attila@inf.unideb.hu

Abstract: Mathability in its definition refers to cognitive infocommunication and combines machine and human cognitive capabilities essential for mathematics. In the paper educational aspects of the notion are considered. A new proposal of learning outcomes taxonomy is presented.

Keywords: Mathability; Education; Constructive Learning; Taxonomy of Learning Objectives

\section{Introduction}

In the age of a technological revolution one can easily find various devices supporting problem solving in general. Among others, making decisions, statistical inferences, complicated calculations, as well as deriving formulas can be listed. These devices equipped with adequate applications provide an aid both for further development of sciences and for everyday education. It is reasonable to classify these smart machines in order to make immediate decisions in choosing which one to use for a given task or to estimate the level of human abilities the machine operator must have to use it successfully. Such an attitude towards research and education influences habits of self-education as well as modern teaching methods. Additionally, when taking into consideration the changing perceptive templates of the younger generation it is worthwhile to investigate new learning methods aided with the described devices.

In the paper we present an overview of three aspects. First of all, we discuss the idea of mathability which refers to devices with high mathematical and logical potential. Next, cognitive patterns are considered from a point of view of 
constructive educational methods. Finally, a proposal of a new taxonomy of learning outcomes and educational goals is demonstrated.

Referring to the definition of cognitive infocommunications (CogInfoCom; cf. [1] and [2]), we describe how people can communicate with machines to possess new knowledge. Moreover, we contribute to cognitive sciences by investigating patterns of young people's perceptions and their methods of assimilating new information, building their knowledge system with problem solving and experience, using devices equipped with applications of high level of mathability. We show how a cognitive process in education co-evolves with infocommunication devices. We also give evidence that the human brain may interact with the capabilities of systems which support cognition.

\section{The Concept of Mathability}

In the educational literature, the notion of mathability is interpreted as human mathematical ability. A broader idea of the concept was introduced in the paper [3] (cf., also, [2]). Mathability was defined as any combination of artificial and natural cognitive capabilities relevant to mathematics. Hence, it is an object of investigation of cognitive infocommunications. The range of its interest stretches from low-level arithmetic operations to high-level symbolic reasoning.

Connected to mathability, in papers [7], [16] and [17], examples of computeraided solutions of mathematical problems were presented. In [7], symbolic calculations and computer algebraic methods were used to derive the solutions of linear functional equations with a computer program (cf., also, [14] and [15]), while in [16] and [17], an animation related to a generalized convexity concept was described. Education aspects of mathability were also investigated by several authors (cf., e.g., [5], [9], [10], [11]).

In article [9], it was pointed out that a quantification of artificial mathematical capabilities would be useful. For instance, contemporary educational institutions allow the use of calculators or other mobile equipment to solve mathematical tasks not only during classes but also during formal examinations. Having a kind of measure for the mathability level, it could be precisely determined which level of mathability a tool should have. Given an official mathability level on the tools, it would be very easy to check it even during or right before any exam. It is natural to ask:

1) what sort of a smart device is allowed to be applied,

2) who and in which form should control whether the device does not exceed the admissible capabilities. Given a mathability level officially on the tools, it would be very easy to check it even during or right before any exam. 


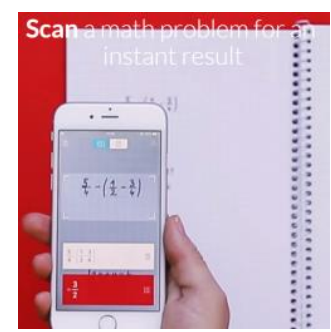

Figure 1

Easy solution with Photomat

It should be taken into account that powerful tools are easily accessible and extensively applied. Machines like popular smart phones provide access to symbolic and algebraic methods. Let us mention a simple mobile application called Photomat (see Figure 1, https://photomath.net/en/). After scanning any handwritten formula, equation, etc., it returns a result. Moreover, it is possible to observe each consecutive step of the solution.

Such a wealth of smart devices and Internet applications combined with characteristics of the younger generation should be taken into consideration while investigating modern education. In further chapters we will try to meet the challenge.

\section{Contemporary Cognition Patterns}

\subsection{Foundation of Constructive Education}

By constructive education we mean building knowledge with creating both new notions or algorithms and relations between the notions through experience. The idea came up a long time ago but nowadays it is becoming meaningful. It is noteworthy that J. Dewey's approach to school education which, in his opinion, should present real life problems and students should be given a chance to experiment. Experiential education consisting of experience, experiments, freedom as well as goal-oriented learning resulted in the concept of progressive education [13]. Among other fathers of constructivism Gy. Pólya [22], J. Bruner [8] and J. Piaget [21] should be mentioned since they influenced the method of problem solving thinking and contemporary progressive education, which is so useful in mathematics, techniques, informatics and other sciences.

For further reading it is worth mentioning D. Kolb's thesis dated from 1976 and referring to learning styles and experiential learning, too [19]. Namely, D Kolb and R. Fry presented a model of an experiential learning cycle (see Figure 2) built of four elements: concrete experience, observation and reflection, forming abstract 
concepts and testing the concepts in new situations [20]. The authors point out that a student can start learning at any of the four steps and then follow the cycle. The starting point is chosen according to the student's learning style.

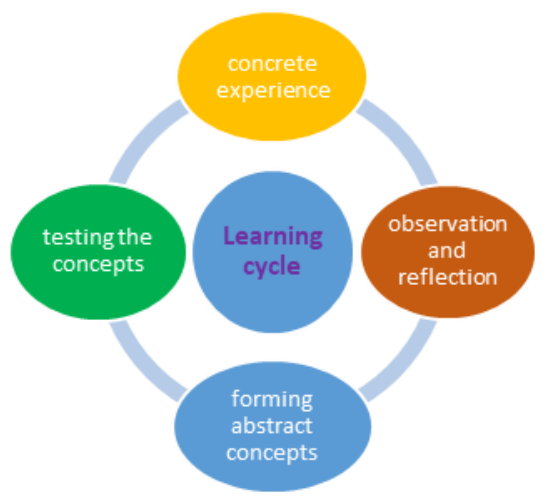

Figure 2

Kolb's experiential learning cycle

Kolb and Fry's learning styles inventory was topped off with identifying four styles:

- converger, who is good at practical application of ideas, hence the converger can start the cycle with abstract conceptualization and immediate active experimenting,

- diverger, who generates ideas and can see things from various perspectives, so the diverger would rather start the cycle with concrete experience and reflective observation,

- assimilator, who is able to create theoretical models and inductive reasoning, the assimilator would choose abstract conceptualization and reflective observation,

- accommodator, who is skillful in doing things, solves problems intuitively and immediately, takes a risk, the accommodator focuses on concrete experience and active experimentation.

The idea of progressive education will be the foundation of building a taxonomy of learning outcomes which we discuss in Chapter 4.

\subsection{Contemporary Cognitive Templates}

New modern multimedia devices seem to be overused by teenagers. On the other hand, they have worked out a habit of searching for keywords and immediate matching, comparing and concluding. 
A short example can help to compare the efficiency of reading between teenagers and people aged $40+$. A text with a mathematical problem was given both to a teacher of mathematics (aged 40+) and an ordinary student (aged 17). The text contained an imperfection which led to a false solution. Hence, it was necessary to find the error in the content. The teacher and the student decided to browse the Internet to find a description of a similar problem. Among a group of similar texts only one fitted the discussed problem completely. It was the student who found it first, compared it with the original task and pointed out the imperfection. While the teacher was still analyzing the content the student compared only keywords which in his opinion were crucial for the correct description of the problem. This simple situation showed us that the process of transforming data is different for people trained in working with traditional (printed) texts and those who explore hypertexts (cf. [10]; for further information, we refer to [18]).

Of course, this is not the only difference creating an educational generation gap. Some more aspects are presented in the following part. The style of reading as well as other cognitive templates described below provide assumptions for building a new idea of education aided with high mathability level devices, which is part of the cognitive infocommunication area.

\subsubsection{Young Generation Habits}

In the papers [9] and [10], among others, ways of self-education based on Internet sources of knowledge were investigated. Some risks were pointed out, when the learning process is not controlled or misses an essential stage.

To prove this, three groups (students of mathematics, students of informatics, lower secondary pupils trained in problem solving) were given mathematical or programming problems to solve. The majority of them failed to find the solutions. It clearly shows that in some cases constructive methods of self-education end in failure. Namely, there were 3 main procedural errors observed.

First of all, the majority of students used a short Wikipedia explanation which was only an introduction to the detailed description. They did not get to the essence of the required algorithm since they did not spend enough time to read the full explanation. Moreover, the tasks - learning by discovering - had the greatest influence on their knowledge. Although they were given further explanation, during the exam they recalled mainly the part they discovered on their own (read more in [10]).

Secondly, we observed how misleading it is to read keywords when they fit the students' prior knowledge systems. For instance, having a task to code some numbers with Fibonacci coding, students found (again only in Wikipedia) a short definition explaining that "Fibonacci coding is a universal positional code which encodes positive integers into binary code words. It is one example of representations of integers based on Fibonacci numbers." (cf. [10]). Students did not read the explanation further since they were sure they had understood the 
definition. Most of them neither considered substantial details nor examined any samples. As a result, for instance, in order to code the number 47 some students: (1) applied binary coding, (2) used Fibonacci numbers, hence they obtained:

$$
47=101111_{2}=\mathrm{F} 1+\mathrm{F} 2+\mathrm{F} 3+\mathrm{F} 4+\mathrm{F} 6=1+2+3+5+13=24 .
$$

Finally, superficiality turned out to be a general problem. Students limited the source of their knowledge to Wikipedia. They were satisfied with a sketchy solution and did not find it worthwhile to understand the core of the problem and its solution nor to reflect on the obtained result.

On the other hand, young people are able to use mobile devices to support their calculus. In [9] we presented, among others, an example of the use of Wolfram Alpha (https://www.wolframalpha.com) to analyze derivatives of the first and second order when the students' aim was to investigate properties of a given function and to draw its graph. Surprisingly, it was the student with the least mathematical competences who solved the problem correctly and finished the task in the shortest time. Aiding calculation and deriving formulas with the mentioned application on-line, he obtained partial solutions immediately and interpreted the results properly.

This brief characterization of young people efficiently searching for keywords, being contented with sketchy solutions but well trained in concluding and matching new knowledge with their prior knowledge system, capably using smart devices to support necessary calculus, should be supplemented with the young people's habit of overusing multimedia. As an immediate result they are less patient, and more often give up solving a task when it seems to be too difficult. It should be mentioned that, our observation's show that, the average time for a teenager to focus on a single complex problem has significantly shrunk. All the features above influence young people's perception, knowledge assimilation, educational practices and learning capabilities. For further investigations of this topic, we refer to the paper [4].

Our aim is to start adapting educational methods to reflect the above characterization. First, it is essential to determine the necessary and sufficient foundation of sciences. Then, we should find how much the foundation should be known and how deep it should be understood before mathability devices are efficiently applied to solve problems and construct new knowledge. To clarify the idea, we present the following example. It shows that the scientist does not need to know a complicated calculation of an advanced multivariable analysis method to understand and use its results.

\subsubsection{Advanced Statistics Research Example}

Let us consider a case of using a statistical data mining engine where algorithms for finding a solution are unknown to an ordinary user. Although the algorithms are hardly understandable for their authors, they give phenomenal solutions. 
Having general knowledge about time series and statistical forecasting we used SAS $^{\circledR}$ Enterprise Miner ${ }^{\mathrm{TM}}$ to analyze road events in Bydgoszcz between 2002 and 2007 in order to forecast the number and severity of road events and accidents in the city for a short period of time in 2008 . We chose 8 out of 17 attributes of road events (location on the road net, human related reasons, number of people killed, number of people injured, etc.). There were altogether 37372 records to consider.

The application we used requires building a visual project combining tools and methods of data preparation and transformation like in Figure 3 (https://www.sas.com/en_gb/software/enterprise-miner.html). As a result, we obtained tables abundant with numbers (see Figure 4). Then, it was enough to interpret them and draw conclusions.

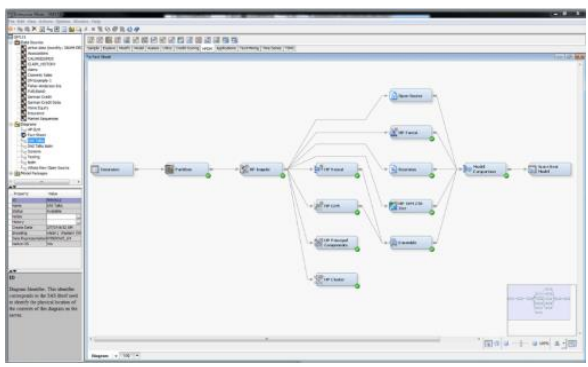

Figure 3

Visual project of statistical analysis with SAS ${ }^{\circledR}$ Enterprise Miner $^{\mathrm{TM}}$
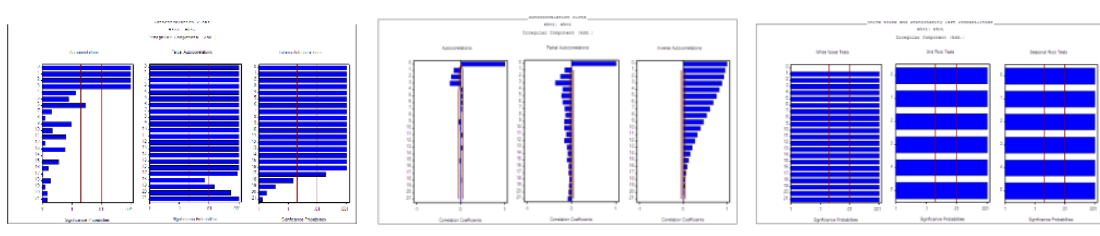

Figure 4

Analysis of irregular components - autocorrelation indicators, white noise tests, unit roots tests

It was not necessary to understand, for instance, the method of imputation of missing data; we only had to know which method of imputation fits best the set of data. Again, it was not needed to know how the thousands of values are processed in time series analysis. As researches we had to define the dependent variables, the set of independent variables, mark a type of model supposed to describe dependencies between variables and chose a plan of investigation, for instance, imputation, analyzing white noise, analyzing autocorrelation order, checking whether the process is stationary, etc. Applying a trial and error method we found that the model of seasonal exponential smoothing fits best the given data, the model is stationary with autocorrelation of order two. Although the R-square coefficient of determination was only 0.148 (which proves that the goodness of fit of the model was very weak) we used the model for further prediction. In the research it was crucial to set the parameters of the model and understand the 
obtained values, namely to know how they should be interpreted. Only some particular definitions had to be known and understood to build an advanced model of forecasting. It was not required to feel fluent in multivariable statistical analysis to use the achieved model for forecasting.

For further examples related to databases, in which the problem appears how to define necessary and sufficient knowledge for applying ICT successfully, we refer to $[12]$.

\section{Mathability in Education}

As far as school education is concerned usually computer aided methods refer to Internet sources of knowledge or to the use of applications supporting the teachers' job. Such programs as Cabri and Geogebra are popular and often used in primary and secondary schools. Academic didactics is aided rather by Wolfram Mathematica, Statistica, MathLab and computer-aided design (CAD) systems.

Here, we would like to highlight three aspects of implementing computer aided mentoring into the everyday life of school pupils (for more details we refer to [10]).

Discovering. Let us consider an example of designating the sum of a convergent series. The notion, on the regular basis, is introduced to students in the first year of sciences. However, it can be easily assimilated by students of upper secondary school. The basic question is: is it necessary to know the formal definition to use a computer application and find the required sum? Using Wolfram Alpha we have an immediate result (see Figure 5, https://www.wolframalpha.com).

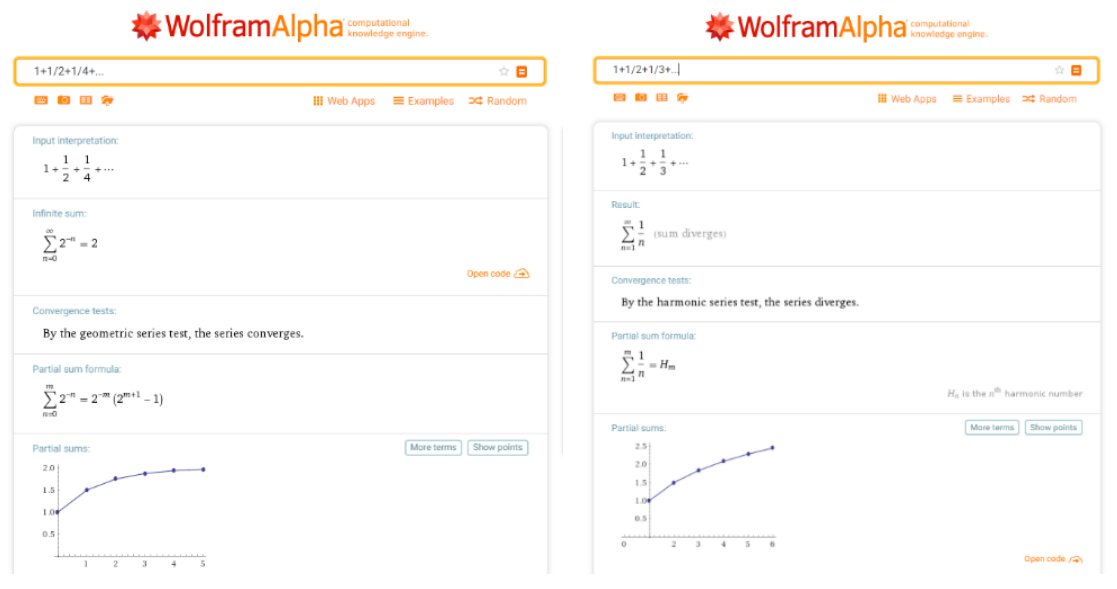

Figure 5

Infinite sum in Wolfram Alpha 
Students can learn that an infinite sum, for instance $1+1 / 2+1 / 4+1 / 8+\ldots$, can have a finite value. On the other hand, a similar infinite sum $1+1 / 2+1 / 3+1 / 4+\ldots$ results in a message "sum diverges". Of, course we do not need any knowledge to produce the results, but it is important to understand what distinguishes one task from the other. Hence, it is worthwhile to understand the mechanism of series convergence.

Students need to know and understand the notion of a sequence limit, which was the essential knowledge necessary to solve the problem. Then, Wolfram Mathematica or another application can be used to compute consecutive partial sums of the above series and represent the obtained values on graphs. Students can easily observe that the sequence of partial sums of the first series is convergent and in the other case - it is not. The students can remark that the finite limit of the sequence of partial sums is equal to the sum of the series. The students succeeded to formulate a convergence criterion on their own.

Interpretation. Let us consider another example of introducing academic knowledge to students of upper secondary school. Namely, the students learned the notions of Taylor series and Taylor approximation of a function. Next, as an exercise they approximated a function $f(x)=\sin x$ with Maclaurin series, obtaining

$\sin (x)=x-\frac{x^{3}}{3 !}+\frac{x^{5}}{5 !}-\ldots$

In order to interpret the results they applied Wolfram Mathematica again to observe simultaneously the graphs of both functions from equation (1), i.e. the graph of the sin function and graphs of polynomials of various ranks. Then, students concluded that a trigonometric function can be locally represented as a simple polynomial function.

Proofs. Finally, it is worthwhile to comment how much mathability devices are applicable in investigations where commonly used methods fail. For instance, students of a technical major were asked to examine the existence of a local extremum at the point $P(0,0)$ for two functions:

$$
\begin{aligned}
& f(x)=x^{4}-y^{4}, \\
& f(x)=(x+y)^{4}-(x-y)^{4} .
\end{aligned}
$$

The standard method of finding the determinant of partial derivatives of the second order was not applicable since both determinants were equal to zero. In such a case representing the functions graphically enabled students to prove that the function given by formula (2) has no extremum at $P(0,0)$ since in any neighborhood of $P$ there exists a point for which the value of the function is positive and there exists a point for which the value of the function is negative. Analogously, they proved that the function given by formula (3) has a local minimum at $P$. 
In the above-mentioned examples the students were guided by their mentors. The problem arises when the students must do a task without mentoring, simply using any source of knowledge or any useful mathability device. Then, the strategy should be changed and the habit of asking questions comes in handy provided that it follows a well ordered structure of questioning. For more details, we refer to [11]. First, we will describe examples of learning outcomes taxonomies in order to draw a theoretical background for creating such a questioning structure.

\section{Taxonomies of Learning Outcomes}

\subsection{Classical Taxonomy}

The classification of educational objectives named after Bloom is one of the most commonly used taxonomies [6]. Let us recall his model of cognitive (knowledgebased) domain consisting of six stages:

1) remembering,

2) comprehending,

3) applying,

4) analyzing,

5) synthesizing,

6) evaluating.

The model is compatible with Kolb's cycle where Kolb's concrete experience corresponds to Bloom's remembering, comprehending and applying, observation and reflection corresponds to analyzing, forming abstract concepts - to synthesizing which, in fact, is adequate for building new knowledge, finally testing the concepts in new situations corresponds to evaluating which ends the cycle. From that point we start the cycle again, on a higher level.

Bloom suggested that the process of learning started with gaining knowledge, then students had to understand it, apply it in typical situations, analyze it before they were able to use it in new or problematic cases. Evaluating completed the process of creating new notions and methods before they could be applied as a base for further learning. This is why Bloom's concept is suitable for traditional, direct teaching. As opposed to this, Kolb states it is possible to start a learning process from an arbitrary stage according to the student's learning style preferences (described in Chapter 2). Moreover, Bloom's model was created over 60 years ago when the sources of knowledge were limited. Easy access to informative data bases, and the above described characterization of cognitive behaviors enable us to build a new pattern of computer assisted education. 


\subsection{Proposal of a Taxonomy for Constructive Education Aided with High Level Mathability Devices}

In our papers [9], [10] we pointed out that working with multimedia sources of knowledge requires paying more attention to the selection and assessment of the gathered information. On the other hand, we have already stressed how important it is to reflect on the results obtained while learning by trial and error. Selfeducation with broad informative data bases is often followed by a lack of reflection over the obtained false results or misunderstanding caused by superficiality. We also gave examples of how useful computer aided mentoring can be. Now, following the mentor-related methods, we would like to present a scheme of cognitive learning objectives adjusted to constructive methods supported with smart devices, compatible both with Bloom's and Kolb's systems. We will compare the common elements of the three models.

First, let us consider a cognitive model of using informative data bases (like the Internet, multimedia, etc.) to learn or create new knowledge (Table 1).

We will consider both constructive teaching methods based on knowledge gained by students on their own and methods of non-mentored constructive learning. Assuming that students gather information by themselves, the first step should be to browse knowledge sources, search for information and already solved examples. The step is consistent with Bloom's remembering stage since young people do not feel the need to memorize information if it is so easily accessible on the net. In Kolb's cycle we could compare it to the stage of concrete experience.

The second step is unfortunately frequently omitted by students. However, it is extremely important from the point of view of building a solid foundation for further education. This is the stage of evaluating and selecting information that fits the prior knowledge system, is understandable (not too complicated) and credible. Two Blooms stages are consistent with this step: analyzing and comprehending. It also corresponds to Kolb's observation and reflection stage.

In the next step, students assimilate the new knowledge into the prior knowledge system, build analogies, find relations, and draw conclusions. If they have learned an algorithm they can try their own computation with other data or in similar cases. This is what corresponds to Bloom's applying and Kolb's testing the concept.

Having gained some new experience students interpret new knowledge or, according to Bloom, synthesize it. Finally, similarly to Blooms's model, they reflect on an overall result, evaluate new knowledge or methods. The last two stages can be compared to Kolb's forming abstract concepts. From that point the cycle starts again from the beginning since new questions should arise and make students search for more information and new methods. 
Table 1

Comparison of computer aided education, Bloom's taxonomy and Kolb's cycle for knowledge

\begin{tabular}{|c|c|c|c|}
\hline Level & $\begin{array}{l}\text { Computer aided } \\
\text { education }\end{array}$ & Bloom's taxonomy & Kolb's cycle \\
\hline \multirow{3}{*}{$\begin{array}{l}\text { Knowledge } \\
\text { and } \\
\text { understanding }\end{array}$} & $\begin{array}{l}\text { Browsing, Searching, } \\
\text { Sample usage }\end{array}$ & Remembering & $\begin{array}{l}\text { Concrete } \\
\text { experience }\end{array}$ \\
\hline & \multirow{2}{*}{$\begin{array}{l}\text { Evaluation } \\
\text { of information, } \\
\text { Selection, }\end{array}$} & Analyzing & \multirow{2}{*}{$\begin{array}{l}\text { Observation and } \\
\text { reflection }\end{array}$} \\
\hline & & Comprehending & \\
\hline \multirow[t]{4}{*}{ Abilities } & $\begin{array}{l}\text { Assimilating into } \\
\text { prior knowledge }\end{array}$ & \multirow[t]{2}{*}{ Applying } & \multirow[t]{2}{*}{ Testing the concept } \\
\hline & $\begin{array}{l}\text { Applying, } \\
\text { Own computation }\end{array}$ & & \\
\hline & Interpreting & Synthesizing & \multirow{2}{*}{$\begin{array}{l}\text { Forming abstract } \\
\text { concepts }\end{array}$} \\
\hline & $\begin{array}{l}\text { Reflecting Evaluating } \\
\text { results }\end{array}$ & Evaluating & \\
\hline
\end{tabular}

Taking into consideration that easy access to high mathability level devices gives a chance for learning by doing as well as for learning by trial and error, it is reasonable to propose a similar pattern for learning or creating new methods, procedures or algorithms (Table 2).

In this case we assume that first of all students have a theoretical foundation which does not necessarily mean that they are acquainted with the formal definitions or know and understand details of the appropriate theorems. This is why it is important to establish a necessary and sufficient level of knowledge for such a foundation. The stage of remembering and understanding the foundation corresponds to two first steps of Bloom's model: remembering and comprehending. However, understanding refers only to the proper knowledge base.

Next, students choose and apply an appropriate mathability device or application in order to compute results, derive formulas or obtain other required solutions. It corresponds to Bloom's stage of applying. The first two steps correspond to Kolb's concrete experience.

Then Bloom's analyzing and Kolb's observation and reflection stages come, which in our model means interpreting the obtained results. Students assess the accuracy and correctness of the results, check their accordance with assumptions, formulate interpretation of the quantities they achieved, etc. Again, this is a frequently omitted step in a student's work even if it is substantial.

Now, students have the expertise to do their own computation in different, sometimes problematic cases what Kolb calls testing the concept and corresponds to Bloom's synthesizing stage. 
Eventually, students reflect on the result, which is a possessed new ability, algorithm or procedure. They try to think of a new use of the result, evaluate its usefulness, find its limitations, etc. This stage corresponds to evaluating and forming abstract concepts in Bloom's and Kolb's models, respectively.

It is reasonable to divide both classifications (for knowledge and abilities) into two parts: 1) knowledge and its comprehension, 2) abilities or mathabilities. Such a division is consistent with former existing models. It should be mentioned that abilities in the division refer to mental abilities such as assimilating, applying (mentally), interpreting, reflecting, evaluating, while mathability refers to the ability of applying smart devices for further reasoning.

Table 2

Comparison of a computer aided education, Bloom's taxonomy and Kolb's cycle for abilities

\begin{tabular}{|l||l|l|l|}
\hline \multicolumn{1}{|c||}{ Level } & Computer aided education & $\begin{array}{l}\text { Levels of Bloom's } \\
\text { taxonomy }\end{array}$ & Kolb's cycle \\
\hline $\begin{array}{l}\text { Knowledge } \\
\text { and } \\
\text { understanding }\end{array}$ & $\begin{array}{l}\text { Remembering and } \\
\text { understanding the } \\
\text { foundation }\end{array}$ & $\begin{array}{l}\text { Remembering } \\
\text { Comprehending } \\
\text { foundation }\end{array}$ & $\begin{array}{l}\text { Concrete } \\
\text { experience }\end{array}$ \\
\cline { 1 - 3 } Mathability & $\begin{array}{l}\text { Computing aided with } \\
\text { smart devices }\end{array}$ & Applying \\
\hline \hline \multirow{2}{*}{ Abilities } & Interpreting results & Analysing & $\begin{array}{l}\text { Observation and } \\
\text { reflection }\end{array}$ \\
\cline { 2 - 4 } & Own computing & Synthesizing & $\begin{array}{l}\text { Testing the } \\
\text { concept }\end{array}$ \\
\cline { 2 - 4 } & $\begin{array}{l}\text { Reflection }= \\
\text { Evaluation of results }\end{array}$ & $\begin{array}{l}\text { Forming abstract } \\
\text { concepts }\end{array}$ \\
\hline
\end{tabular}

\section{Conclusions}

Perception and learning practices have been influenced by common habits of using multimedia, modern tools of cognitive infocommunication and facilities for gathering information using instant searching for keywords. Hence, the ways of human cognition and knowledge assimilation have been modified. Modern mathematical, technical and science education should fit the new habits and capabilities of young people. Applying high level mathability devices and applications as well as using multimedia knowledge sources, guided by mentors can be very helpful. Thanks to such methods the presented characteristic of the young generation, e.g. lack of accuracy, sketchy solutions, lack of assessment and reflection, could be easily eliminated.

\section{Acknowledgement}

This work was supported by the construction EFOP-3.6.3-VEKOP-16-201700002. The project was co-financed by the Hungarian Government and the European Social Fund. 
This research was also supported by the Hungarian Scientific Research Fund (OTKA) Grant K-111651 and by Kazimierz Wielki University in Bydgoszcz.

The research described in this paper was partially performed in the Virtual Reality Laboratory of the Faculty of Informatics of the University of Debrecen, Hungary.

The authors are thankful to the anonymous reviewers for their valuable comments.

\section{References}

[1] Baranyi P., Csapó Á.: Definition and Synergies of Cognitive Infocommunications, Acta Polytechnica Hungarica, 9:67-83, 2012

[2] Baranyi P., Csapó Á., Sallai Gy.: Cognitive Infocommunications (CogInfoCom) Springer, 2015

[3] Baranyi P., Gilányi A.: Mathability: Emulating and Enhancing Human Mathematical Capabilities, $4^{\text {th }}$ IEEE Conference on Cognitive Infocommunications (CogInfoCom) IEEE, 2013, 555-558

[4] Biró P., Csernoch M.: Deep and Surface Metacognitive Processes in NonTraditional Programming Tasks, $5^{\text {th }}$ IEEE Conference on Cognitive Infocommunications (CogInfoCom) IEEE, 2014, 49-54

[5] Biró P., Csernoch M.: The Mathability of Computer Problem Solving Approaches, $6^{\text {th }}$ IEEE Conference on Cognitive Infocommunications (CogInfoCom) IEEE, 2015, 111-114

[6] Bloom B. S.: Taxonomy of Educational Objectives: The Classification of Educational Goals, Susan Fauer Company, Inc., 1956

[7] Borus G. Gy., Gilányi A.: Solving Systems of Linear Functional Equations with Computer, $4^{\text {th }}$ IEEE Conference on Cognitive Infocommunications (CogInfoCom) IEEE, 2013, 559-562

[8] Bruner J. S., Haste H.: Making Sense. The Child's Construction of the World, Methuen, New York, 1987

[9] Chmielewska K., Gilányi A.: Mathability and Computer-aided Mathematical Education, in $6^{\text {th }}$ IEEE Conference on Cognitive Infocommunications (CogInfoCom) IEEE, 2015, 473-477

[10] Chmielewska K., Gilányi A., Łukasiewicz A.: Mathability and Mathematical Cognition, in $7^{\text {th }}$ IEEE Conference on Cognitive Infocommunications (CogInfoCom) IEEE, 2016, 245-250

[11] Chmielewska K., Matuszak D.: Mathability and Coaching, in $8^{\text {th }}$ IEEE Conference on Cognitive Infocommunications (CogInfoCom) IEEE, 2017, 427-431

[12] Csernoch, M., Dani, E.: Data-Structure Validator: An Application of the HY-DE Model, $8^{\text {th }}$ IEEE Conference on Cognitive Infocommunications (CogInfoCom) IEEE, 2017, 197-202 
[13] Dewey J.: Experience and Education, NY Kappa Delta Pi, New York, 1938

[14] Gilányi A.: Characterization of Monomial Functions and Solution of Functional Equations Using Computers (Charakterisierung von monomialen Funktionen und Lösung von Funktionalgleichungen mit Computern, German), PhD Thesis, University of Karlsruhe, 1995

[15] Gilányi A.: Solving Linear Functional Equations with Computer, Math. Pannon., 9:57-70, 1998

[16] Gilányi A., Merentes N., Quintero R.: Mathability and an Animation Related to a Convex-like Property, $7^{\text {th }}$ IEEE Conference on Cognitive Infocommunications (CogInfoCom) IEEE, 2016, 227-231

[17] Gilányi A., Merentes N., Quintero R., Presentation of an Animation of the m-convex Hull of Sets, $7^{\text {th }}$ IEEE Conference on Cognitive Infocommunications (CogInfoCom) IEEE, 2016, 307-308

[18] Kirschner P. A., De Bruyckere P.: The Myths of the Digital Native and the Multitasker, Teaching and Teacher Education 67:135-142, 2017

[19] Kolb D. A.: The Learning Style Inventory: Technical Manual, McBer, Boston, 1976

[20] Kolb D. A., Fry, R.: Toward an Applied Theory of Experiential Learning, in C. Cooper (ed.) Theories of Group Process, John Wiley, London, 1975

[21] Piaget J.: Studies in Child Psychology (Studia z psychologii dziecka, Polish), PWN, Warsaw, 1966

[22] Pólya Gy.: How to Solve It, Princeton University Press, Princeton, 1945 